Research in Astronomy and Astrophysics manuscript no.

(LTEX: ms128.tex; printed on October 26, 2018; 8:46)

\title{
Interaction between Granulation and Small-Scale Magnetic Flux Observed by Hinode
}

\author{
Jun Zhang, Shuhong Yang and Chunlan Jin \\ Key Laboratory of Solar Activity, National Astronomical Observatories, Chinese Academy of Sciences, \\ Beijing 100012, China; zjun@ourstar.bao.ac.cn
}

\begin{abstract}
With the polarimetric observations obtained by the Spectro-Polarimeter on board Hinode, we study the relationship between granular development and magnetic field evolution in the quiet Sun. 6 typical cases are displayed to exhibit interaction between granules and magnetic elements, and we have obtained the following results. (1) A granule develops centrosymmetrically when no magnetic flux emerges within the granular cell. (2) A granule develops and splits noncentrosymmetrically while flux emerges at an outer part of the granular cell. (3) Magnetic flux emergence as a cluster of mixed polarities is detected at the position of a granule as soon as the granule breaks up. (4) A dipole emerges accompanying with the development of a granule, and the two elements of the dipole root in the adjacent intergranular lanes and face each other across the granule. Advected by the horizontal granular motion, the positive element of the dipole then cancels with pre-existing negative flux. (5) Flux cancellation also takes place between a positive element, which is advected by granular flow, and its surrounding negative flux. (6) While magnetic flux cancellation takes place at a granular cell, the granule shrinks and then disappears. (7) Horizontal magnetic fields enhance at the places where dipoles emerge and where opposite polarities cancel with each other, but only the horizontal fields between the dipolar elements point orderly from the positive element to the negative one. Our results reveal that granules and small-scale magnetic flux influence each other. Granular flow advects magnetic flux, and magnetic flux evolution suppresses granular development. There exist extremely large Doppler blue-shifts at the site of one cancelling magnetic element. This phenomenon may be caused by the upward flow produced by magnetic reconnection below the photosphere.
\end{abstract}

Key words: Sun: granulation — Sun: magnetic fields — Sun: photosphere — techniques: polarimetric

\section{INTRODUCTION}

Both observations and simulations reveal that granules and small-scale magnetic elements spread all over 
granules excluding the surrounding dark lanes amounts to $1^{\prime \prime} .1$ (Namba \& Diemel 1969), or 1".35 (Bray et al. 1984), while the mean cell size of the granular elements including one-half of the surrounding dark lanes is $1^{\prime \prime} .94$ (Bray \& Loughhead 1977), or $1^{\prime \prime} .76$ (Roudier \& Muller 1986). Frequently the granules expand and split into smaller components that drift apart, and the fragments may turn grow and fragment, or merge with others, or shrink and decompose.

Magnetic fields in the quiet Sun can be classified into three categories based on their locations and morphologies: network (Leighton et al. 1962), intranetwork (IN; Livingston \& Harvey 1975) and ephemeral regions (Harvey \& Martin 1973). The network elements are confined to the supergranular boundaries and the IN ones locate within the supergranular cells. The spatial distribution and time evolution of IN magnetic features are closely associated with the solar granulation (Lin \& Rimmele 1999). Small flux and size with rapid time changes make the IN field difficult to observe and characterize (Keller et al. 1994). However, much progress has been made in IN morphology dynamics and some quantitative aspects, such as flux distribution (Wang et al. 1995), lifetime (Zhang et al. 1998a), mean horizontal velocity field (Wang et al. 1996; Zhang et al. 1998b), motion pattern and evolution (Zhang et al. 1998b, c, 2006).

The magnetic flux emergence seems to be significantly influenced by the granular motion. The research about the horizontal IN fields suggests that small magnetic loops are being advected toward the surface by the convective upward motion of the plasma inside the granules (Lites, et al. 1996; Orozco Suárez et al. 2008). The horizontal motion inside the granules carries the vertical magnetic flux toward the intergranular lanes (Harvey et al. 2007; Centeno et al. 2007). Then most of these IN magnetic elements are destroyed by three mechanisms: merging with IN or network elements of the same polarity, cancellation of opposite polarity elements, or separation and disappearance at the position where they appear (Zhang et al. 1998a). Furthermore, the magnetic emergence also has important influence on the shape of the underlying granulation pattern leading to the so-called "abnormal granulation” (Cheung et al. 2007).

Using the continuum intensities, vector magnetic fields and Doppler velocities derived from the Stokes profiles obtained by the Spectro-Polarimeter (SP; Lites et al. 2001) on board Hinode (Kosugi et al. 2007), we mainly study the relationship between the development of granular structures and the emergence and cancellation of small-scale (with a typical size of $\sim 1^{\prime \prime}$ in this paper) magnetic elements from their birth to death. In Sect. 2, we describe the observations and the strategy of Stokes profile inversion. Then we present the relationship between granular development and magnetic flux emergence (in Sect. 3) and cancellation (in Sect. 4). The conclusions and discussion are all given in Sect. 5.

\section{OBSERVATIONS AND INVERSION STRATEGY}

The SP instrument of the Solar Optical Telescope (SOT; Ichimoto et al. 2008; Shimizu et al. 2008; Suematsu et al. 2008; Tsuneta et al. 2008) aboard Hinode provides the full Stokes profiles of two Fe lines at 630.15 $\mathrm{nm}\left(g_{e f f}=1.67\right)$ and $630.25 \mathrm{~nm}\left(g_{e f f}=2.5\right)$ in four modes (fast map, dynamics, normal map and deep magnetogram). In order to investigate both the structure and the evolution of granules and magnetic elements, the observational field-of-view (FOV) should not be too small and the cadence should be high enough. So we adopt the data taken from 11:33 UT to 17:51 UT on June 1, 2007 in the fast map mode. 
Sun region near disk center $\left(-6^{\prime \prime},-199^{\prime \prime}\right)$ with a FOV of $8^{\prime \prime} .86 \times 162^{\prime \prime} .3$. The scan step (X direction in Fig. 1 ) is $0^{\prime \prime} .295$, and the pixel sampling along the slit direction (Y direction in Fig. 1) is $0^{\prime \prime} .32$. The integration time for each slit was $3.2 \mathrm{~s}$ with a noise level in the polarization continuum of $1.4 \times 10^{-3} I_{c}$.

By using the inversion techniques based on the assumption of Miline-Eddington atmosphere model (Yokoyama 2008, in preparation), we can derive vector magnetic fields from the full Stokes profiles. Although the inversion procedures encounter difficulties in convergence toward and uniqueness of the solutions when confronting with noisy profiles (Lites et al. 2008), they will be largely independent of the noise and the field strength initialization if only the pixels with polarization signals above a reasonable threshold are inverted (Orozco Suárez et al. 2007). Here we only analyze the pixels with total polarization degrees above 1 time of the noise level in the polarization continuum in order to exclude some profiles that cannot be inverted reliably.

Values of 13 free parameters are returned from the inversion, including the three components of magnetic field (field strength $B$, inclination angle $\gamma$, azimuth angle $\phi$ ), the stray light fraction $\alpha$, the Doppler velocity $V_{l o s}$, and so on. Since the pair of Fe I lines in low flux quiet Sun regions are not capable of distinguishing between the intrinsic magnetic field and the filling factor (Martínez González et al. 2006), the flux density is a more appropriate quantity to describe. Here, we show the equivalent, spatially resolved vector magnetic fields by "apparent flux density" of the longitudinal and transverse components (Lites et al. 1999), i.e. $B_{a p p}^{L}=(1-\alpha) B \cos \gamma$ and $B_{a p p}^{T}=(1-\alpha)^{1 / 2} B \sin \gamma$, respectively. The longitudinal component $B_{a p p}^{L}$ may be considered as the magnitude of the line-of-sight (LOS) component of a spatially resolved magnetic field that produces the circular polarization signal as the observed signal, and the transverse component $B_{a p p}^{T}$ is vertical to the LOS that would produce the observed linear polarization signal. In the vector field measurements based on the Zeeman Effect, there exists 180 degree ambiguity in determining the field azimuth. Potential field approximation is one of the fairly acceptable methods to resolve the ambiguity (Wang 1999). The Doppler velocities, which are evaluated from the center of the Stokes I profiles according to Fe 630.25 $\mathrm{nm}$ line and averaged over the whole FOV, are well defined even in weak field regions (Chae et al. 2004).

Figure 1 shows the whole FOV images retrieved from the SP data obtained from 14:29:54 UT to 14:31:51 UT. They are continuum intensity map, corresponding longitudinal magnetogram, transverse magnetogram, and Dopplergram from left to right, respectively. The zero value areas in the magnetograms represent the pixels not included in the analysis because of their low polarization signals. Windows $2-7$ in Fig. 1 outline the corresponding FOVs of Figs. 2-7, respectively. Windows 4 and 6 locate near network magnetic fields, while others at IN fields.

\section{RELATIONSHIP BETWEEN GRANULAR DEVELOPMENT AND MAGNETIC FLUX EMERGENCE}

In order to explore the relationship between granular development and magnetic flux emergence in the quiet Sun, we examine time sequence of continuum intensity maps, corresponding vector magnetograms and Dopplergrams. We find that granules and magnetic elements influence each other in complicated ways. 
It appears that a granular structure develops centrosymmetrically when no magnetic flux emerges within the granular cell, just as shown in Fig. 2. The contour curves outline the granule focused to study with continuum intensity ratio $I_{c} / I_{0}=1.03$, where $I_{0}$ represents the average continuum intensity in the whole FOV. From 14:25 UT on, the granule expanded continuously with a mean apparent horizontal velocity of $1.5 \mathrm{~km} \mathrm{~s}^{-1}$ (calculated along the direction of arrow "1"). At 14:29 UT, a dark core appeared near the granular center and expanded larger. Comparing the maps of continuum intensity with the Dopplergrams, we can see that the granular cell always suffered Doppler blue-shifts during their development process.

We have also noticed that a granular structure develops and splits noncentrosymmetrically while magnetic flux emerges within the granular cell. A typical example is exhibited in Fig. 3. Arrows "1" and "2" denote two negative elements emerging orderly at an outer part of the granule within 6 minutes, and their maximum longitudinal apparent flux density reached $100 \mathrm{Mx} \mathrm{cm}^{-2}$. Different from the granular shape shown in Fig. 2, the boundary of this granule became concave as the magnetic flux emerged. At 14:50 UT, the granule split into several small fragments.

Figure 4 shows that lots of magnetic elements emerged as a cluster of mixed polarities while a granule broke up. The granule first appeared at 14:46 UT, then developed gradually and became larger with a mean apparent horizontal expanding velocity of $1.6 \mathrm{~km} \mathrm{~s}^{-1}$ along the direction indicated by the arrow. The main body of the granule split at 15:00 UT; meanwhile the magnetic flux was detected emerging as a cluster of mixed polarities at the position the initial granule located (defined by the ellipses in the longitudinal magnetograms), and reached its maximum absolute value of $4.2 \times 10^{18} \mathrm{Mx}$ at 15:10 UT.

Observations also indicate that the granular motion has significant influence on the magnetic flux emergence. As shown in the first two columns of Fig. 5, a dipole emerged accompanying with the development of a granule. A pair of arrows labelled "2" denote the two magnetic elements of the dipole. At 16:12 UT, the positive element of the dipole appeared and the transverse fields had generally ordered directions (shown by thin arrows in the transverse magnetograms at 16:12 UT). At this time, the granular region suffered larger Doppler blue-shifts with a mean velocity of $-1.8 \mathrm{~km} \mathrm{~s}^{-1}$. Two minutes later, the other element of the dipole appeared and the transverse fields between the dipolar elements enhanced with their directions pointing orderly from the positive element to the negative one. The two elements of the dipole then lay on the two sides of the granule, rooting in the adjacent intergranular lanes.

\section{RELATIONSHIP BETWEEN GRANULAR DEVELOPMENT AND MAGNETIC FLUX CANCELLATION}

Magnetic flux cancellation is a main form of flux disappearance in the solar photosphere, but its physical mechanism is not clearly known in detail. As lacking magnetic field observations with high temp-spatial resolution, the behavior of flux cancellation at a sub-arcsec spatial scale has not been well researched.

The last three columns in Fig. 5 exhibit the flux cancellation which occurred between the positive element of the dipole and pre-existing negative flux. The positive element (shown by arrow " 3 ") was advected by the horizontal flow with a mean velocity of $1.7 \mathrm{~km} \mathrm{~s}^{-1}$ along arrow "1" direction towards the pre-existing 
meanwhile strong transverse fields (see the square area) appeared, but their directions were unordered, i.e. with no orientation consistency. By 16:20 UT, the positive element had disappeared completely.

Figure 6 displays a case of magnetic flux cancellation between a positive element (indicated by arrow "1") and its surrounding negative flux. During the growing and splitting process of a granule, the positive element was advected by the granular flow along arrow "2" direction with a mean velocity of $1.0 \mathrm{~km} \mathrm{~s}^{-1}$. At 16:08 UT, it encountered the negative network fields and cancelled with them. Four minutes later, the positive element disappeared totally. Dopplergrams in this figure also show that the granular locations were occupied by the Doppler blue-shift signals.

Besides being advected by granular motion, cancelling magnetic flux also suppresses granular development, as shown in Fig. 7. With the growing process of a granule, a dipole began to emerge at 16:22 UT, and the horizontal magnetic fields between the dipolar elements also appeared, with their directions pointing from the positive element to the negative one, as shown by the arrows in parallelogram "II" region. This dipole was much more obvious at 16:24 UT (shown by a pair of arrows "2"). Before the appearance of dipole "2", there already existed another dipole (denoted by a pair of arrows "1"). From 16:20 UT to 16:26 UT, the pre-existing dipole moved to the newly emerged dipole, with an average velocity of $2.2 \mathrm{~km}$ $\mathrm{s}^{-1}$. The negative element of dipole "1" and the positive element of dipole "2" cancelled since 16:24 UT. Two minutes later, the cancelling negative element split into two segments (denoted by arrows " 3 " and "4", respectively), due to the collision of the cancelling positive element (see arrow "5"). Then the positive element of dipole " 1 " began to move away from the cancelling position along arrow "6" direction with an average velocity of $2.6 \mathrm{~km} \mathrm{~s}^{-1}$, and finally returned to its birth place. The negative element of the newly emerging dipole also moved away from the cancelling position along arrow "7" direction, with an average velocity of $2.5 \mathrm{~km} \mathrm{~s}^{-1}$. At 16:28 UT, segment " 3 " had disappeared, while segment " 4 " and element " 5 " were cancelling violently. At this time, strong transverse fields (inside the parallelogram "III" region) appeared at the cancelling position, but their directions were unordered. At 16:30 UT, the two cancelling elements almost disappeared. From the continuum intensity maps, we can see clearly that the granule shrank rapidly while the magnetic flux cancellation took place. Comparing the longitudinal magnetograms with the corresponding Dopplergrams, we find that, at 16:26 UT, the site of dipolar element " 5 " (outlined by the ellipses) underwent very high Doppler velocity $\left(-3.0 \mathrm{~km} \mathrm{~s}^{-1}\right)$.

\section{CONCLUSIONS AND DISCUSSION}

By examining 6 typical cases, we present in this paper the relationship between emerging (cancelling) smallscale magnetic flux and granular structures in a quiet Sun region near disk center. A granule develops in a centrosymmetric form in the condition that no magnetic flux emerges within the granular cell, and another granular structure develops and splits in a noncentrosymmetrical form while flux emerges at an outer part of the granular cell. As soon as a granule breaks up, magnetic flux emergence as a cluster of mixed polarities appears at the position of the former granule. A dipole emerges accompanying with the development of a granule and cancels with pre-existing flux, due to the advection of the horizontal granular motion. When magnetic flux cancellation takes place at a granular position, the granule shrinks and disappears. Our results 
development. Furthermore, we uncover the evolution of transverse fields and changes of Doppler signals during cancelling process.

Observations show that the horizontal fields of small magnetic loops are advected toward the surface by the upward motion of the plasma inside the granules (Lites et al. 1996) and horizontal motion inside the granules carries the vertical magnetic flux toward the intergranular lanes (Harvey et al. 2007; Centeno et al. 2007). In this paper, the emergence of the dipole connected by horizontal fields which pointed from the positive element to the negative, as shown in Fig. 5, is another case similar to that of Centeno et al. (2007). However, we cannot exclude the possibility that the two elements of dipole "2" spontaneously separated each other under no driving effect of the granular plasma motion when they emerged continuously in an $\Omega$-shaped configuration. Figure 4 shows that magnetic flux emergence as a cluster of mixed polarities at a position where the granule located was detected as soon as the granule split. The split of the granule may result from the emergence of the magnetic flux. There also exists another possibility that the granule split unaffectedly, and then the flux emerged upward by magnetic buoyancy or by convection. In simulation, Cheung et al. (2007) found that the magnetic flux emergence with a longitudinal flux of more than $10^{19} \mathrm{Mx}$ disturbs the granulation, while small-scale flux tubes with less than $10^{18}$ are not sufficiently buoyant to rise coherently against the granulation and produce no visible disturbance in the granules. Our results in this observational study are inconsistent with Cheung et al. (2007), since the emerging magnetic elements with flux lower than $10^{18} \mathrm{Mx}$, e.g. the event displayed in Fig. 3, can also affect the granular development.

2 of the 6 cases indicate that magnetic cancellation was triggered by the pushing effect of the horizontal granular flows (see Figs. 5 and 6). On the other hand, magnetic element also reacted on granules. The example in Fig. 5 reveals that the development of the granule was suppressed by the positive element (denoted by arrow "3"). At 16:20 UT, the element disappeared; meanwhile the granule occupied the element position. Vector magnetic field observations show that the magnetic connection of cancelling elements was changed, and the transverse fields enhanced during the cancelling process (see Figs. 5 and 7). These observations confirm the earlier results that the two components of cancelling magnetic features are initially not connected by transverse fields above the photosphere (Zhang et al. 2001). These results are also consistent with Kubo \& Shimizu (2007), who examined several collision events and found formation of new magnetic connection.

Granules are upwards-moving, hot parcels of gas, exhibiting blue-shifts in the high-resolution spectral image (Nesis et al. 2001). Not unexpectedly in this study, granules always suffered Doppler blue-shifts with an average velocity of $-1.1 \mathrm{~km} \mathrm{~s}^{-1}$. In Fig. 5, the maximum Doppler blue-shift was $-2.0 \mathrm{~km} \mathrm{~s}^{-1}$ at the early emerging stage of dipole " 2 ", while it decreased to $-0.9 \mathrm{~km} \mathrm{~s}^{-1}$ when the dipole well developed. In Fig. 7, the region with Doppler blue-shifts of about $-3.0 \mathrm{~km} \mathrm{~s}^{-1}$ at 16:26 UT is coincident with the site of the cancelling magnetic element " 5 ", which belonged to the newly emerging dipole "2". At the early emerging stage (at 16:20 UT) of this dipole, when the Doppler blue-shifts are expected to be the largest during the dipolar lifetime, the Doppler velocity is around $-2.2 \mathrm{~km} \mathrm{~s}^{-1}$, smaller than that at cancelling stage. We suggest that the excess of the blue-shifts at 16:20 in Fig. 7 are produced by the magnetic flux reconnection below the photosphere, as demonstrated in Fig. 8. When magnetic reconnection occurs at 
directional plasma jets form and eject from the "X-point" along the field lines. If the reconnection takes place below the photosphere, the upward plasma jets (denoted by arrows " 1 " and "2") move across the solar surface from inner to outer, and Doppler blue-shifts will be observed in the photospheric surface. The area where the larger blue-shifts appear is relevant to the topology of magnetic field lines. The magnetic field lines jet "1" moves along are more vertical than that of jet "2", so the blue-shifts appear at the site of one magnetic element (marked by "p"). Chae et al. (2004) reported an example of magnetic flux submergence at the flux cancelling sites. Their observations also revealed that larger downflows were at the cancelling positive magnetic feature instead of at the polarity inversion line. If magnetic fields are observed with lower spatial resolution, small-scale elements cannot be distinguished separately and several elements combine together to form a larger one. At this condition, the large blue-shifts will appear mainly at the adjacent region of the two big cancelling elements (marked by "N" and "P" in Fig. 8). However, we can not rule out the possibility that the large upward velocities at the cancellation area are caused by the emerging $\mathrm{U}$-shaped flux loops (Parker 1984; Lites et al. 1995).

Since our this study is limited to only several cases, we will make a statistical analysis over a large sample of events to examine whether these results are general or not in our next study.

Acknowledgements We thank the Hinode team for providing the data. Hinode is a Japanese mission developed and launched by ISAS/JAXA, with NAOJ as domestic partner and NASA and STFC (UK) as international partners. It is operated by these agencies in co-operation with ESA and NSC (Norway). This work is supported by the National Natural Science Foundations of China (G10573025, 40674081 and 40890161), the CAS Project KJCX2-YW-T04, and the National Basic Research Program of China under grant G2006CB806303.

\section{References}

Bray, R. J. \& Loughhead, R. E. 1977, Sol. Phys., 54, 319

Bray, R. J., Loughhead, R. E., \& Durrant, C. J. 1984, The solar granulation (2nd edition) (Cambridge and New York: Cambridge University Press), 270

Centeno, R., Socas-Navarro, H., Lites, B., et al. 2007, ApJ, 666, L137

Chae, J., Moon, Y., \& Pevtsov, A. A. 2004, ApJ, 602, L65

Cheung, M. C. M., Schüssler, M., \& Moreno-Insertis, F. 2007, A\&A, 467, 703

Harvey, K. L., \& Martin, S. F. 1973, Sol. Phys., 32, 389

Harvey, J. W., Branston, D., Henney, C. J., \& Keller, C. U. 2007, ApJ, 659, 177

Ichimoto, K., Lites, B., Elmore, D., et al. 2008, Sol. Phys., 249, 233

Keller, C. U., Deubner, F. L., Egger, U., et al. 1994, A\&A, 286, 626

Kosugi, T., Matsuzaki, K., Sakao, T., et al. 2007, Sol. Phys., 243, 3

Kubo, M., \& Shimizu, T. 2007, ApJ, 671, 990

Leighton, R. B., Noyes, R. W., \& Simon, G. W. 1962, ApJ, 135, 474

Lin, H., \& Rimmele, T. 1999, ApJ, 514, 448

Lites, B. W., Elmore, D. F., \& Streander, K. V. 2001, in ASP Conf. Series, 236, 33

Lites, B. W., Kubo, M., Socas-Navarroet, H. et al. 2008, ApJ, 672, 1237

Lites, B. W., Leka, K. D., Skumanich, A., et al. 1996, ApJ, 460, 1019

Lites, B. W., Low, B. C., Martinez Pillet, V., et al. 1995, ApJ, 446, 877

Lites, B. W., Rutten, R. J., \& Berger, T. E. 1999, ApJ, 517, 1013

Livingston, W. C., \& Harvey, J. 1975, BAAS, 7, 346

Martínez González, M. J., Collados, M., \& Ruiz Cobo, B. 2006, A\&A, 456, 1159 
Nesis, A., Hammer, R., \& Roth, M. 2001, Sol. Phys., 200, 11

Orozco Suárez, D., Bellot Rubio, L. R., Del Toro Iniesta, J. C., et al. 2007, PASJ, 59, 837

Orozco Suárez, D., Bellot Rubio, L. R., Del Toro Iniesta, J. C., \& Tsuneta, S. 2008, A\&A, 481, L33

Parker, E. N. 1984, ApJ, 280, 423

Roudier, T., \& Muller, R. 1986, Sol. Phys., 107, 11

Shimizu, T., Nagata, S., Tsuneta, S., et al. 2008, Sol. Phys., 249, 221

Suematsu, Y., Tsuneta, S., Ichimoto, K., et al. 2008, Sol. Phys., 249, 197

Tsuneta, S., Ichimoto, K., Katsukawa, Y., et al. 2008, Sol. Phys., 249, 167

Wang, H., Tang, F., Zirin, H., \& Wang, J. 1996, Sol. Phys., 165, 223

Wang, J. 1999, Fundam. Cosmic Phys., 20, 251

Wang, J., Wang, H., Tang, F., et al. 1995, Sol. Phys., 160, 277

Yokoyama, T. 2008, in preparation

Zhang, J., Lin, G., Wang, J., et al. 1998a, Sol. Phys., 178, 245

Zhang, J., Lin, G., Wang, J., Wang, H., \& Zirin, H. 1998c, A\&A, 338, 322

Zhang, J., Ma, J., \& Wang, H. 2006, ApJ, 649, 464

Zhang, J., Wang, J., Deng, Y., \& Wu, D. 2001, ApJ, 548, L99

Zhang, J., Wang, J., Wang, H., \& Zirin, H. 1998b, A\&A, 335, 341 


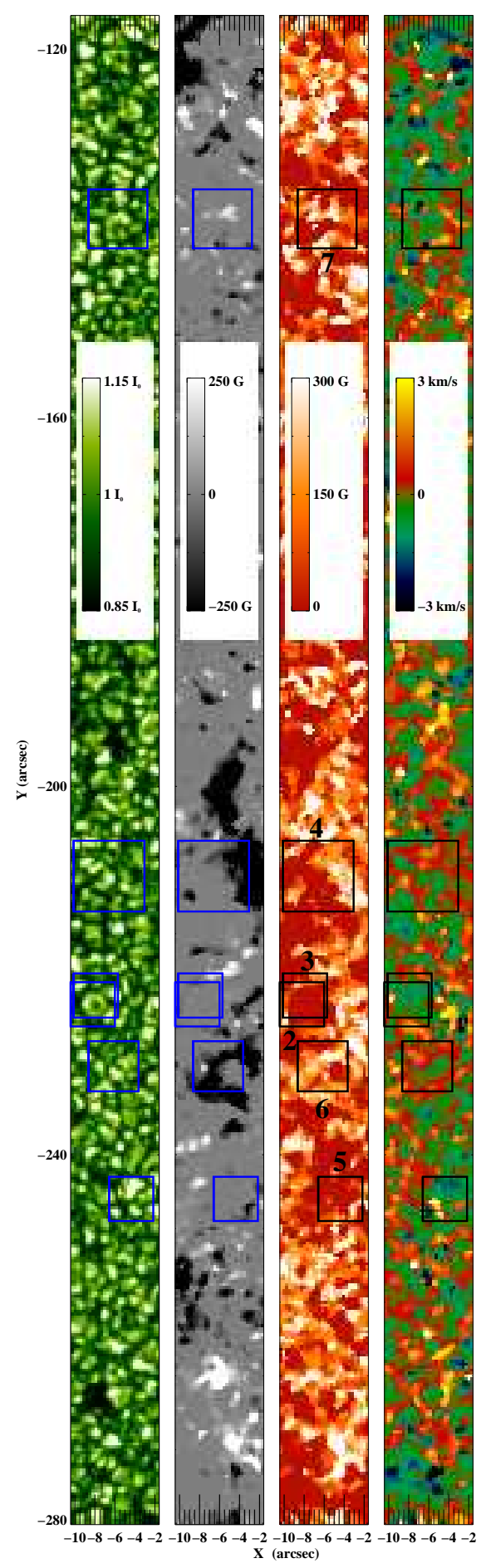

Fig. 1 General appearance of the whole field-of-view (FOV) retrieved from the SP data obtained from 14:29:54 UT to 14:31:51 UT on June 1, 2007. From left to right: continuum intensity map, corresponding longitudinal magnetogram, transverse magnetogram, and Dopplergram. Windows $2-7$ outline the FOVs of Figs. $2-7$, respectively. $I_{0}$ represents the average continuum intensity in the whole FOV. 

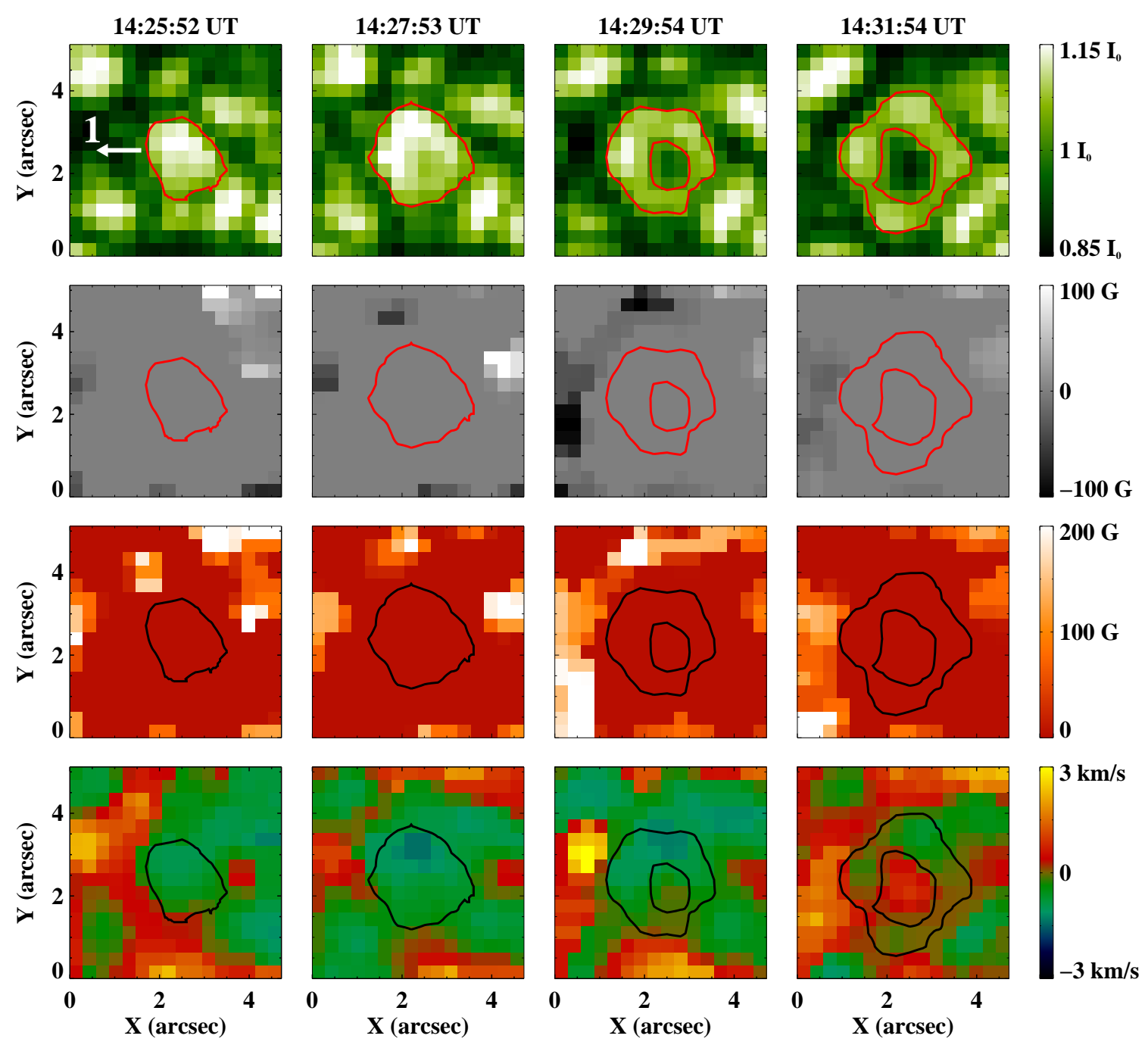

Fig. 2 Temporal evolution of a granule with no magnetic flux emergence. From top to bottom: continuum intensity, corresponding longitudinal field, transverse field and Doppler velocity. The contours in the top row panels represent the granule with continuum intensity $\mathrm{I}_{c} / \mathrm{I}_{0}=1.03$, and they are also overplotted to the corresponding magnetograms and Dopplergrams. Here, only the granule we focus to study is selected to outline. Arrow "1" denotes the direction of the granular flow along which we calculate the apparent horizontal velocity. 

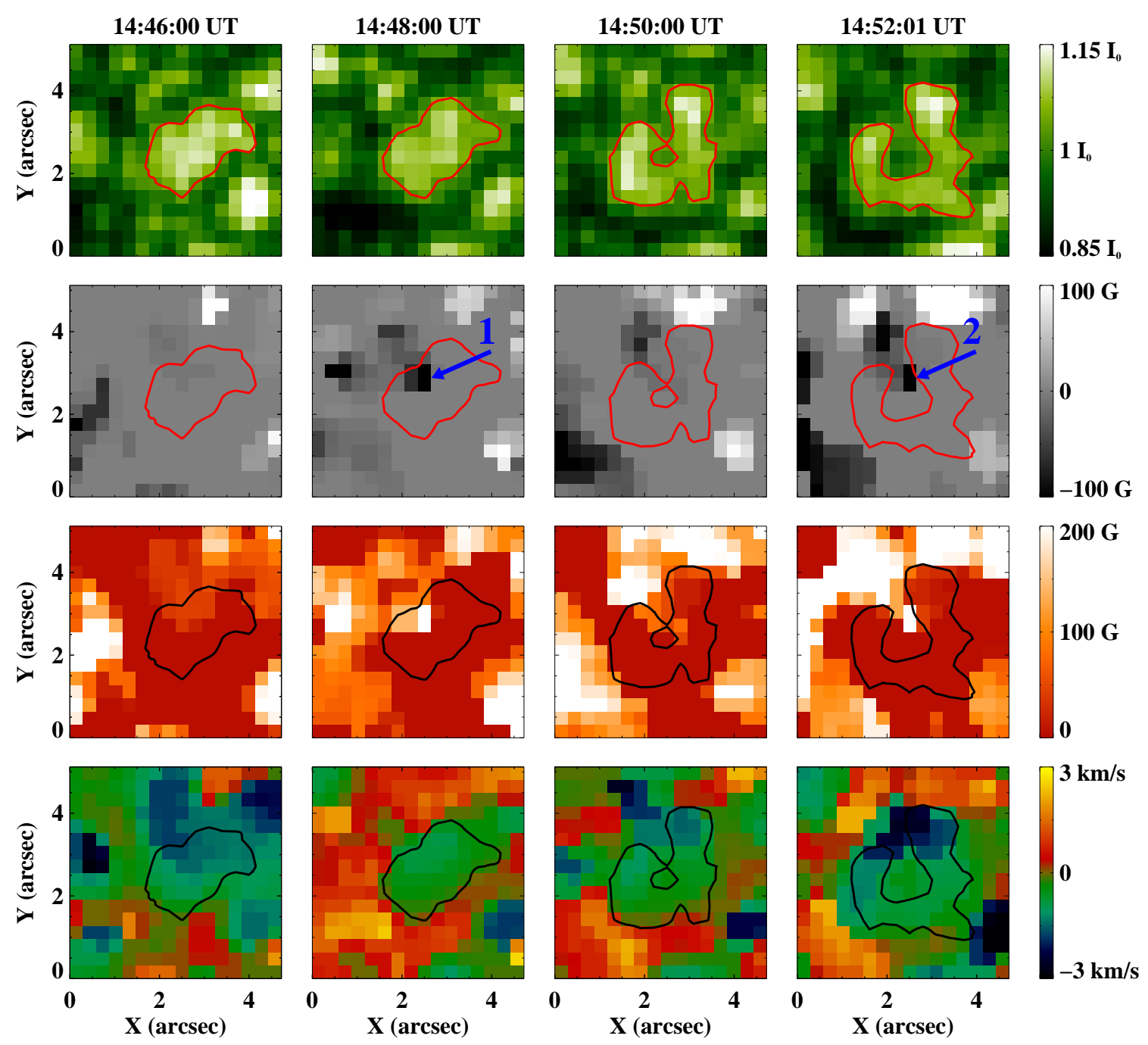

Fig. 3 Similar to Fig. 2 but for a granule accompanying flux emergence. Arrows "1" and "2" denote two magnetic elements emerging orderly at an outer part of the granule. 

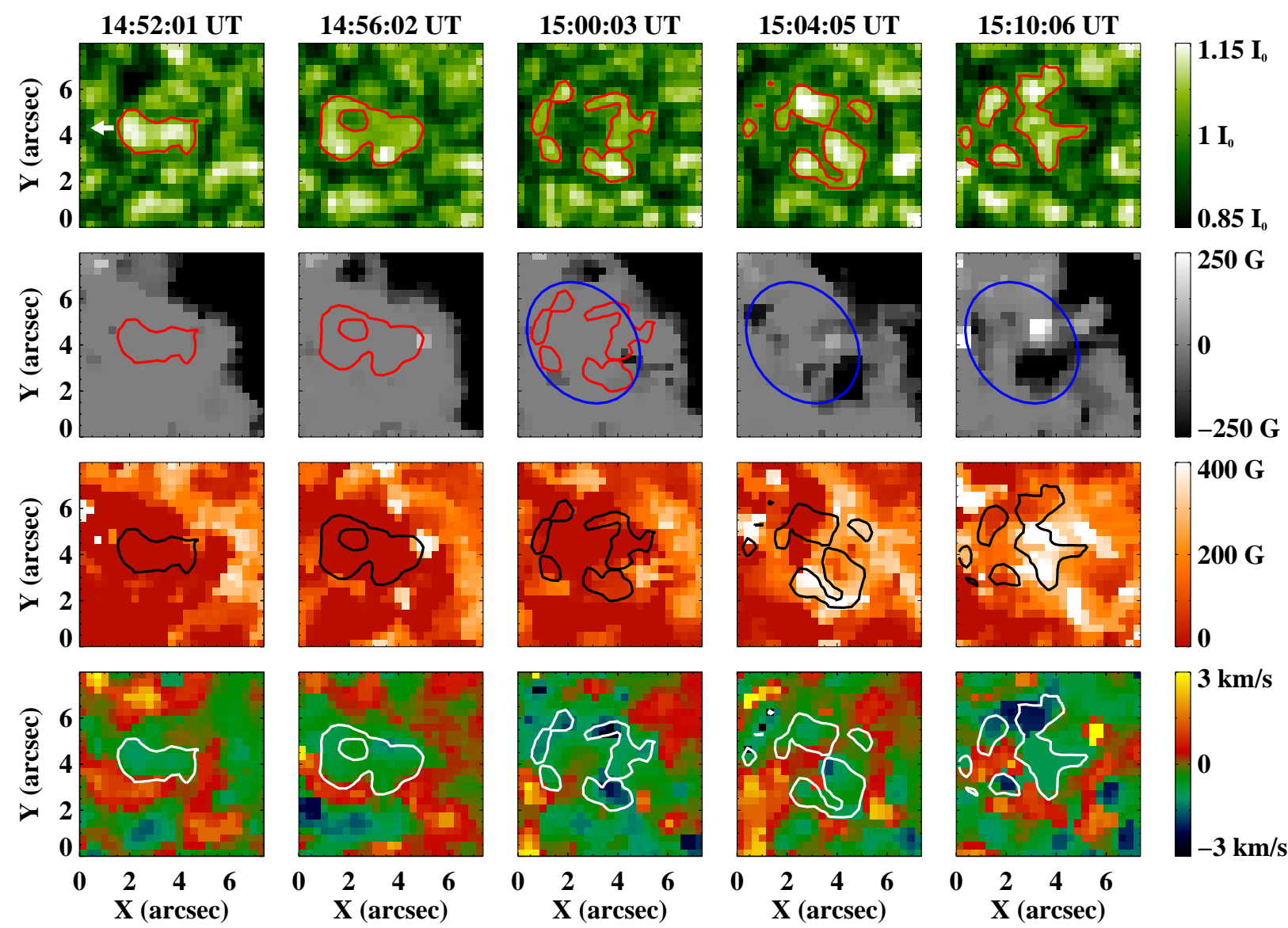

Fig. 4 Similar to Fig. 2 but for magnetic flux emergence as a cluster of mixed polarities following the split of a granule. The ellipses enclose the area where the flux emerges. 

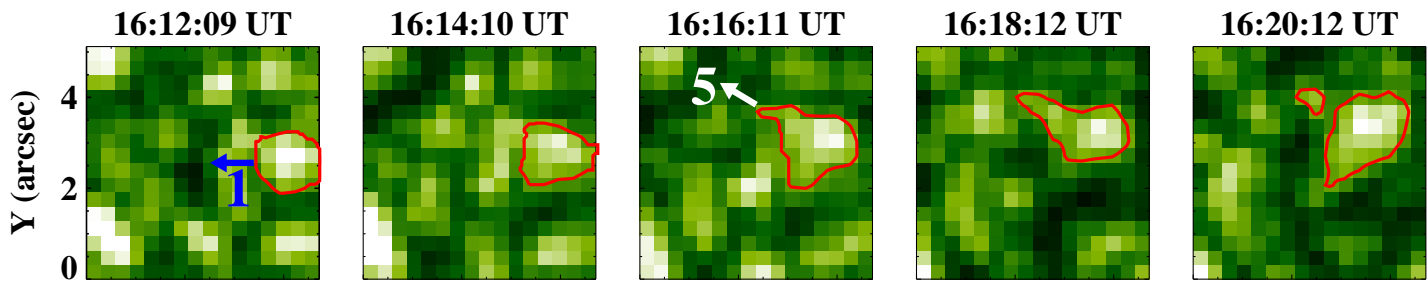

$1.15 I_{0}$
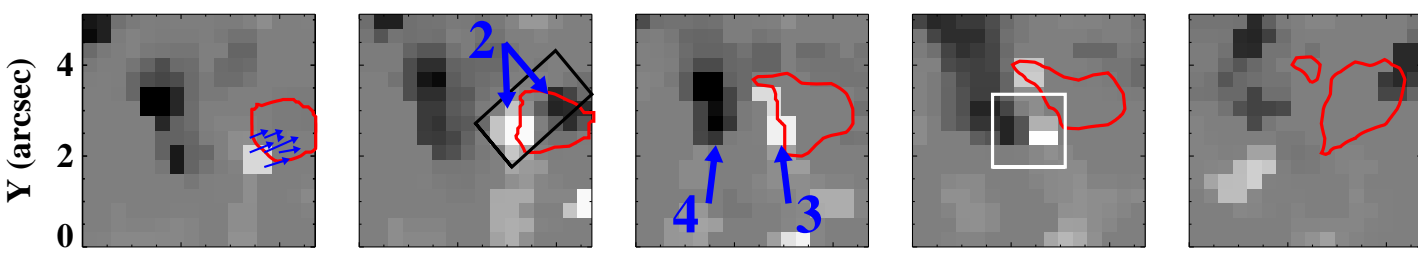

$250 \mathrm{G}$
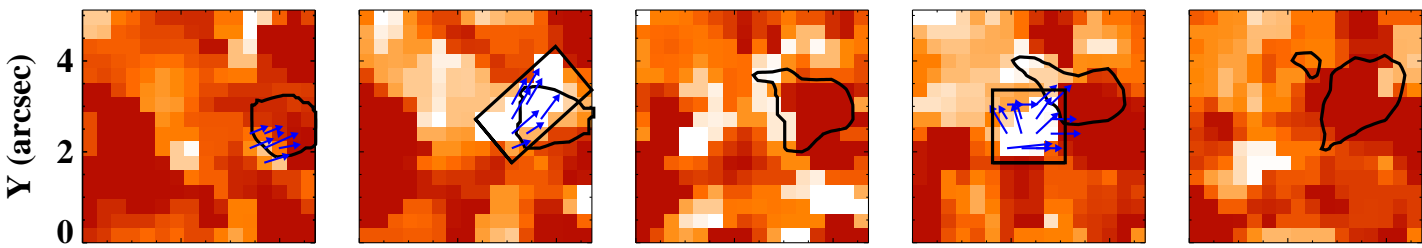

$1 \mathbf{I}_{0}$
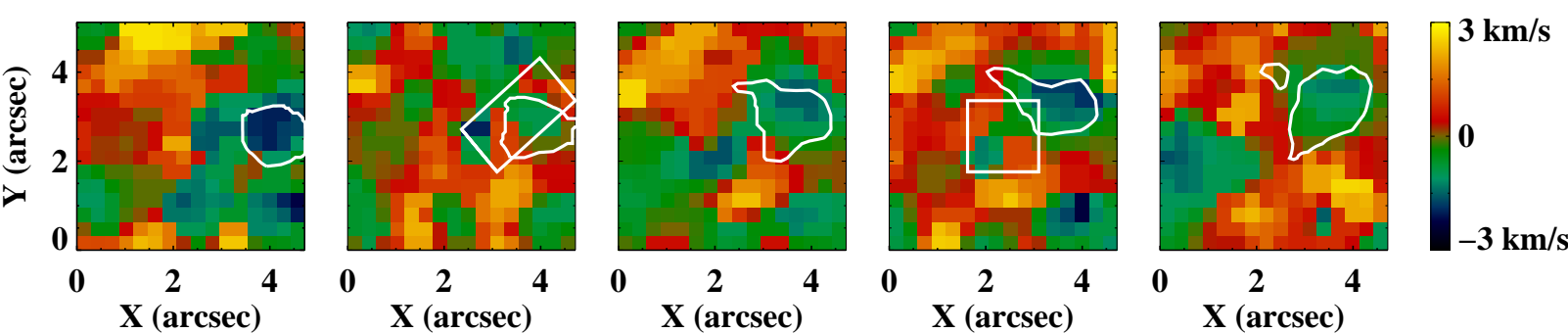

Fig. 5 Similar to Fig. 2 but for emergence of a dipole (first two columns; marked by a pair of arrows "2") and cancellation (last three columns) between a positive element (indicated by arrow "3") belonging to the dipole and a pre-existing negative element (shown with arrow "4"). The parallelograms and squares embody the regions where the transverse fields enhance, and the thin arrows indicate the directions of the local horizontal fields. Arrow "1" denotes the direction along which we calculate the apparent horizontal velocity of the granular flow and arrow " 5 " the direction along which the granule quickly extended. 

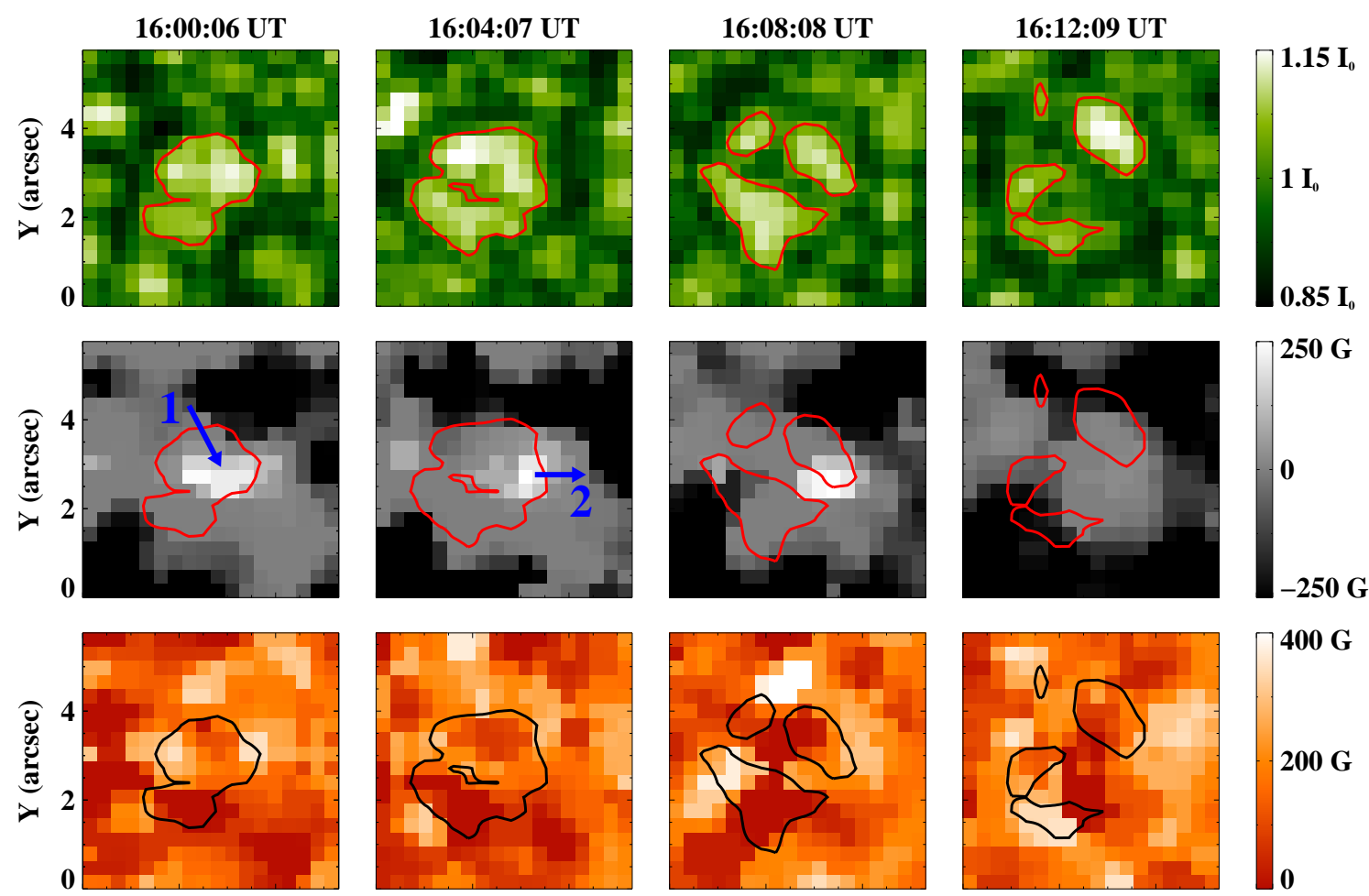

$-250 \mathrm{G}$
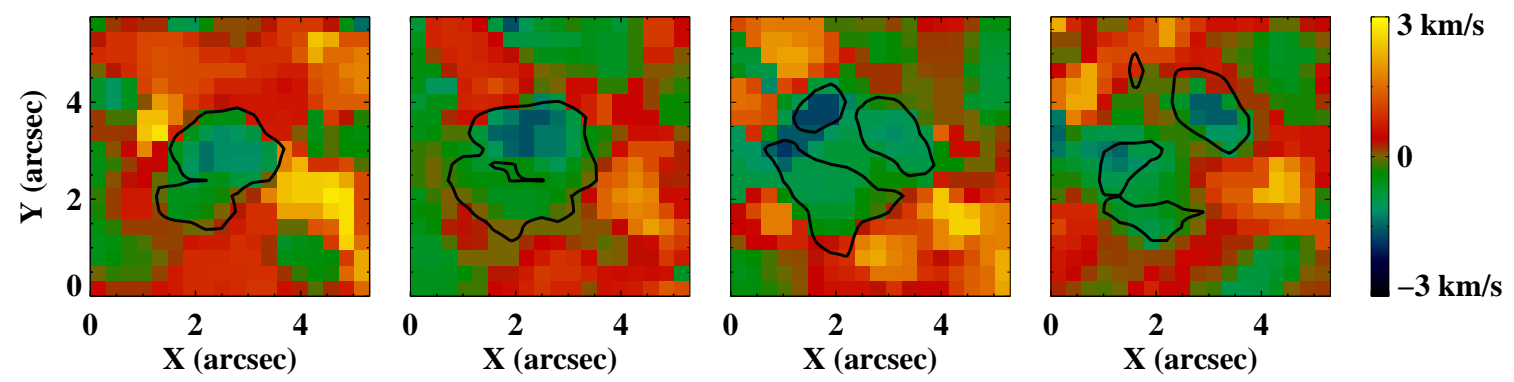

Fig. 6 Similar to Fig. 2 but for flux cancellation of a positive element advected by the granular flow with its surrounding negative flux. Arrow "1" denotes the positive element and arrow "2" moving direction of the element. 

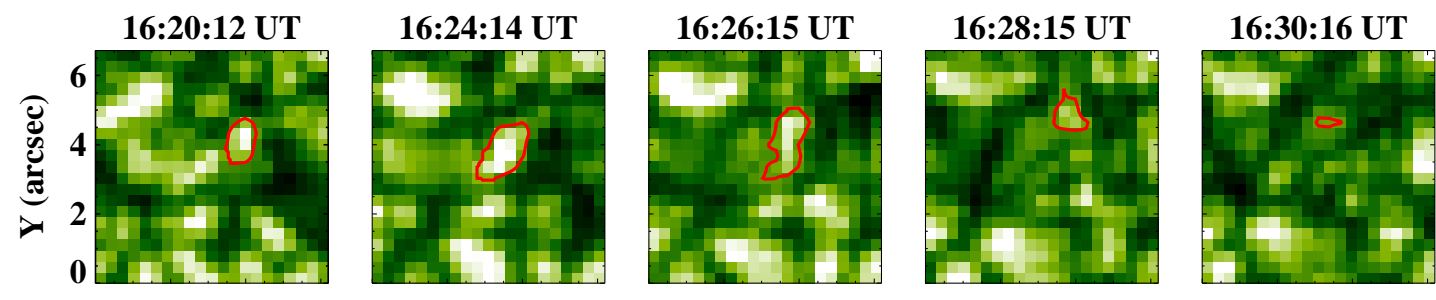

$1.15 \mathbf{I}_{0}$
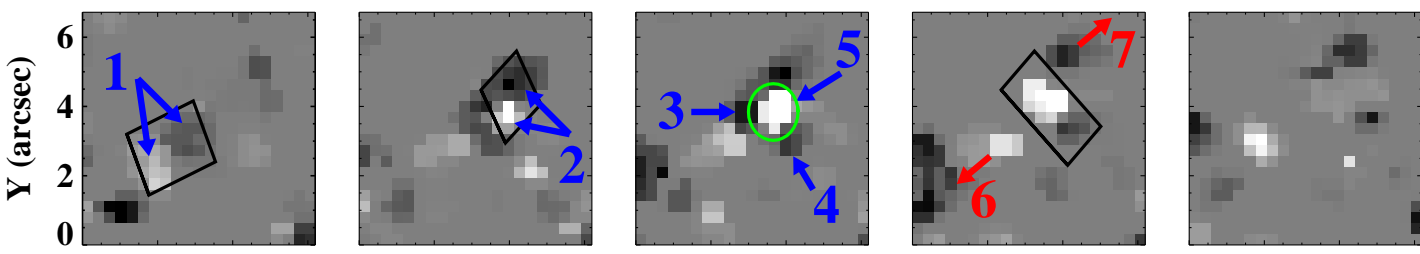

$250 \mathrm{G}$
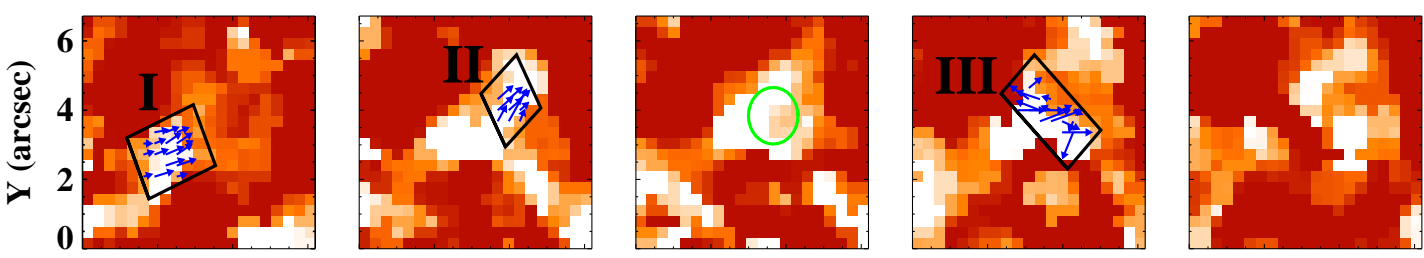

$250 \mathrm{G}$
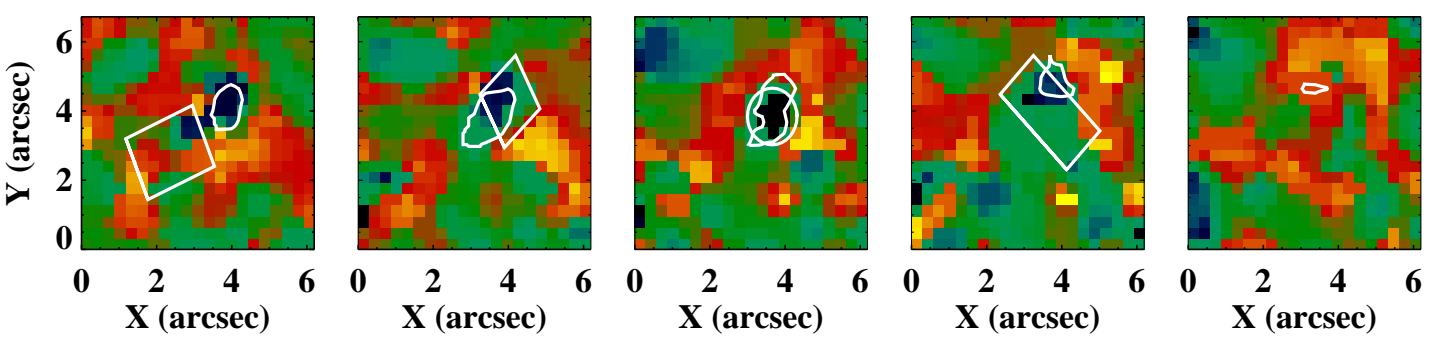

$1 \mathbf{I}_{0}$
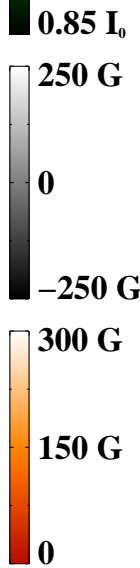

Fig. 7 Similar to Fig. 2 but for shrink process of a granule due to the cancellation between the positive element of a dipole (pointed by a pair of arrows "2") and the negative element of another dipole (denoted by another pair of arrows "1"). Arrows "3" and "4" indicate two segments dividing from the negative element of dipole " 1 ", and arrow " 5 " shows the positive element of dipole "2". Arrows "6" and "7" denote the moving directions of the un-interacting elements of the two dipoles. The parallelograms and the thin arrows within them are same as described in Fig. 5. Note that the arrows in parallelogram "II" are overplotted from the transverse fields at the same location at 16:22 UT. The ellipses mark the position where the remarkable Doppler blue-shifts locate. 


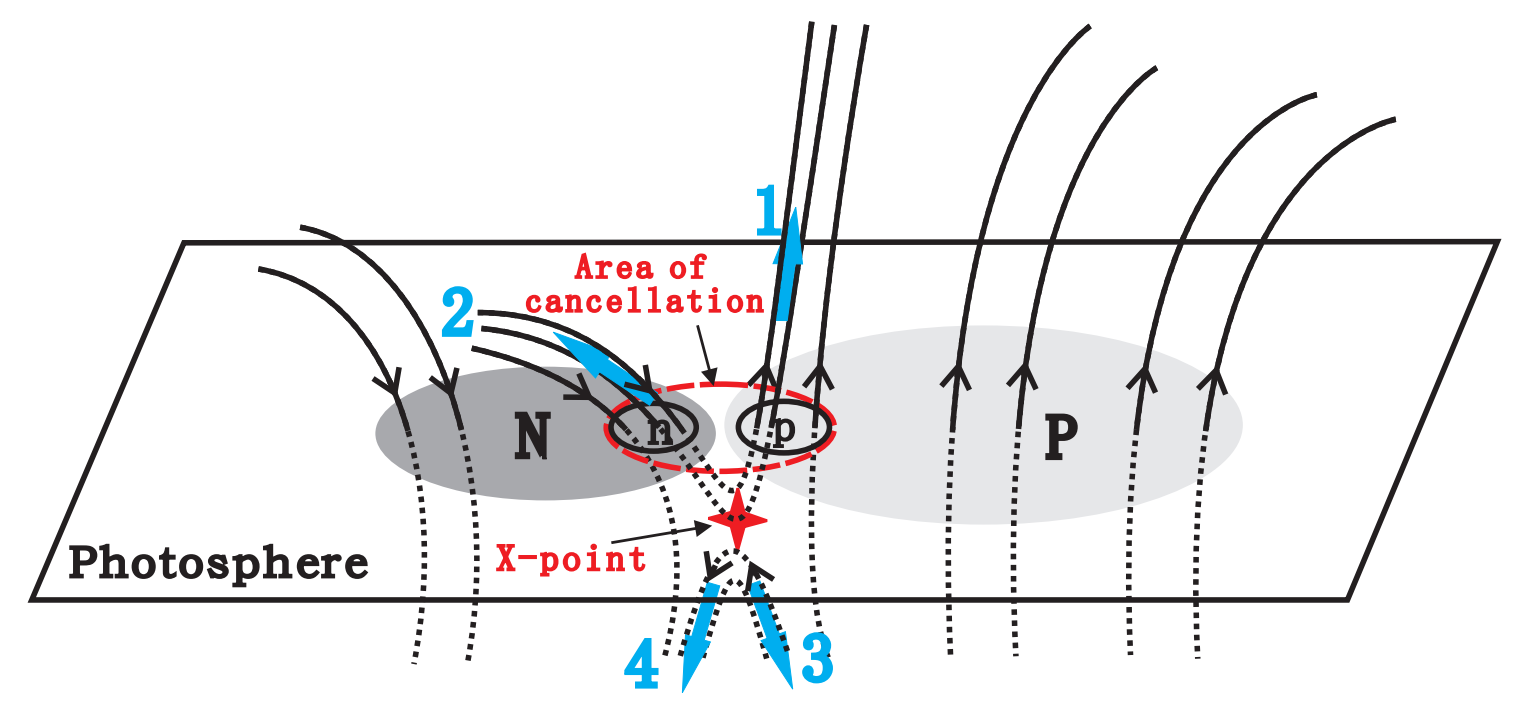

Fig. 8 Sketch illustrating formation of large Doppler blue-shifts locating at one cancelling magnetic element. See the text for details. 